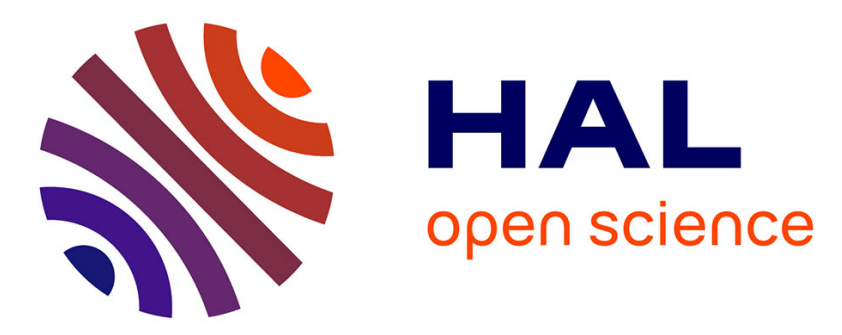

\title{
Modelling of biological decontamination of a water resource in natural environment and related feedback strategies
}

\author{
Sébastien Barbier, Alain Rapaport, Antoine Rousseau
}

\section{To cite this version:}

Sébastien Barbier, Alain Rapaport, Antoine Rousseau. Modelling of biological decontamination of a water resource in natural environment and related feedback strategies. Journal of Scientific Computing, 2016, 68 (3), pp.1267-1280. 10.1007/s10915-016-0178-9 . hal-01138335v4

\section{HAL Id: hal-01138335 \\ https://inria.hal.science/hal-01138335v4}

Submitted on 29 Jan 2016

HAL is a multi-disciplinary open access archive for the deposit and dissemination of scientific research documents, whether they are published or not. The documents may come from teaching and research institutions in France or abroad, or from public or private research centers.
L'archive ouverte pluridisciplinaire HAL, est destinée au dépôt et à la diffusion de documents scientifiques de niveau recherche, publiés ou non, émanant des établissements d'enseignement et de recherche français ou étrangers, des laboratoires publics ou privés. 


\title{
MODELLING OF BIOLOGICAL DECONTAMINATION OF A WATER RESOURCE IN NATURAL ENVIRONMENT AND RELATED FEEDBACK STRATEGIES
}

\author{
S. BARBIER *, A. RAPAPORT ${ }^{\dagger}$, AND A. ROUSSEAU $\ddagger$
}

\begin{abstract}
We show how to combine numerical schemes and calibration of systems of o.d.e. to provide efficient feedback strategies for the biological decontamination of water resources. For natural resources, we retain to introduce any bacteria in the resource and treat it aside preserving a constant volume of the resource at any time. The feedback strategies are derived from the minimal time synthesis of the system of o.d.e.
\end{abstract}

Key words. biological water treatment, hydrodynamics, continuous bioreactors, optimal control, numerical simulations, reduced modelling.

AMS subject classifications. 35Q35, 35Q30, 93A30, 49J15

1. Introduction. Today, mankind is aware that the water resources of our planet are limited. Pollution and over-exploitation of these resources affect many water reservoirs (lakes, phreatic tables, sources or rivers...) on Earth. Preserving the quality of water has thus become a worldwide problem.

The treatment of toxic substances (such as nitrate, insecticides, fungicides...) is usually made by bacterial agents. Typically, micro-organisms are cultivated in tanks or "bioreactors" that are filled with the contaminated water. Well selected bacteria can grow on the pollutant, that is eventually converted into biomass. Then, the separation of biomass from the liquid provides a decontaminated water. These kinds of decontamination are operated in large scale plants dedicated to the treatment of municipal or industrial waste-waters.

The treatment of natural or isolated reservoirs, such as lakes, water-tables or pools, that could have been punctually contaminated, presents some constraints GvD02, SJL ${ }^{+}$07, EPD09. It is usually not allowed to empty the reservoir to be treated in an external station, or to introduce in the resource bacterial agents, that could settle and affect other forms of life (algae, fishes, crustaceans...).

The problem we tackle in this paper concerns the minimization of the treatment duration of such a water resource, that is pumped to be decontaminated in a side bioreactor, and simultaneously pumped back from the output of the reactor through a device of biomass separation (see Figure 2.1). The bioreactor thus operates under a continuous input/output flow so that the volumes of the resource and the reactor are constant at any time.

The operation optimization of fed-batch or continuously-stirred bioreactors has received a great attention in the literature GDG71, DGK72, Alf06, KH00, BBCMA03, Spi04, Alf06, SKLB08. The particularity of the present problem comes from the constraint that no biomass has to be introduced in the contaminated resource.

Recently, this optimization problems has been studied with the help of the theory of optimal control, under simple representations of the spatial inhomogeneity of the

\footnotetext{
*barbier.sebastien22@gmail.com

†UMR INRA SupAgro MISTEA, 2 pl. Viala 34090 Montpellier and EPI MODEMIC, INRIA Sophia-Antipolis Méditerranée, rte de Lucioles, 06902 Sophia-Antipolis, France. alain.rapaport@montpellier.inra.fr

${ }^{\ddagger}$ Inria and Institut de Mathématiques et de Modélisation de Montpellier, Team LEM0N, Bât 5 - CC05 017, 860 rue Saint-Priest, 34095 Montpellier Cedex 5,France. antoine.rousseau@inria.fr
} 
pollutant concentration in the resource GHR11, GRRR12]. These simple (parameterized) models are described in terms of systems of ordinary differential equations in small dimension (one or two), and have allowed to derive the exact optimal feedback strategies.

For large volumes to be treated, for which spatial inhomogeneity of the pollutant concentrations cannot be neglected, one may wonder if these over-simplified spatial representations are satisfactory to describe faithfully the input/output behavior of the pollutant concentration in the resource.

The objective of the present work is similar to Rav00: estimate the quality of the approximations that could be obtained by these simple models, comparing them with accurate simulations of the resource hydrodynamics based on Navier-Stokes equations. The originality of our approach is first to use the feedback strategy that has been determined to be optimal for the simplified model with the accurate model, and secondly to show that calibration of the parameters of the simple model under the feedback control provides a good approximation of the time evolution of the output concentration of pollutant.

Finally, this hybrid combination of numerical simulations of Navier-Stokes models with feedback control of simple models provide bases for future efficient decision making tools.

2. Mathematical modelling: spatial representations. We first present an ordinary differential equations (ODE) model that has been conceived in a purely phenomenological way from the observations of accurate numerical simulations of the hydrodynamics of the lake with the pumps. This model is not a reduced model of the Navier-Stokes equations, but we show that it can satisfactorily approximate the input/output behavior of the lake. Of course, it cannot give any accurate information on the spatial hydrodynamics in-between the input and output locations of the pumps, as a true Navier-Stokes model can do. Nevertheless, we show in Appendix that this model possesses an interpretation, under certain assumptions, in terms of averaged quantities of the solution of the advection diffusion equation of the partial differential equations (PDE) model that we present further in Section 2.2, justifying a posteriori the equations of this ODE model. Its simplicity has allowed us to obtain the optimal policy for the bioremediation based on this model. Subsection 2.1 presents this model and gives the exact expression of the optimal feedback. Then, we show in Subsection 2.2 how the apply this feedback law on a more accurate model with partial derivative equations (PDE) based on the Navier-Stokes equations, in terms of fixed-point operator. As the simple model we propose is parameterized (with two parameters), we finally explain in Subsection 2.3 how we have calibrated those parameters with the simulations of the PDE model coupled with the feedback law.

2.1. ODE-based model and related feedback strategy. We propose a simple representation of the heterogeneity of the pollutant concentration in the water resource with two patches:

1. an "active" patch, where water is pumped out for purification and is reinjected back in the same patch after abatement,

2. a "dead" patch that is supposed to be less directly influenced by advection but merely by passive diffusion with the active one.

This simple two patches model (see Fig 2.1) mimics the observation that one may roughly distinguish two zones that are advection or diffusion dominated. Typically, the active zone contains the shortest current lines that join input from output locations, while the dead one is expected to be away from those lines. 


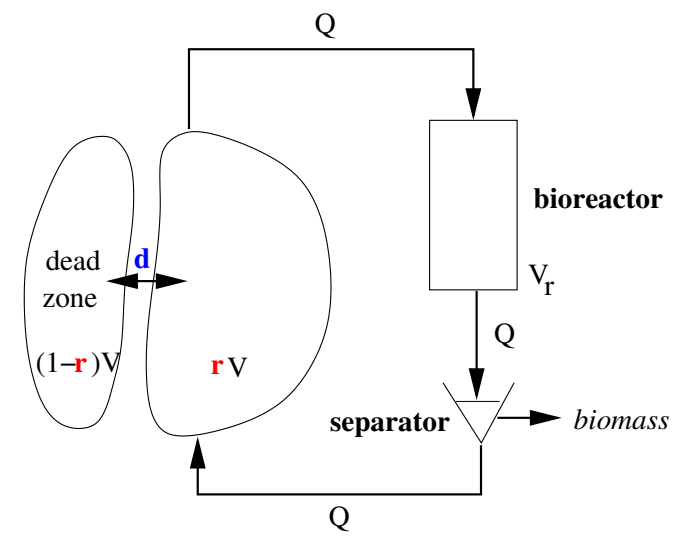

Fig. 2.1: Two patches representation of the resource coupled with the bioreactor, characterized by the parameters $d$ and $r$.
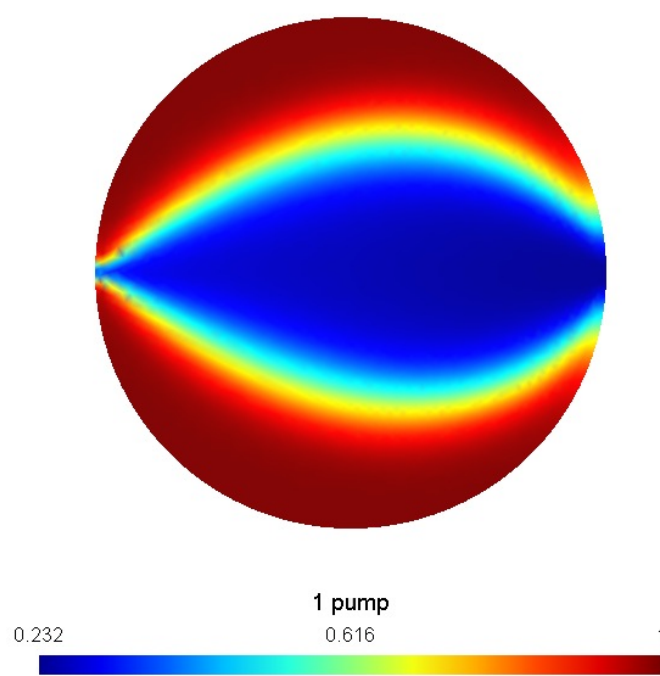

Fig. 2.2: Pollutant concentration in the lake simulated with a PDE-based model given by equations $(2.5)$ to 2.10$)$. The simple model mimics the observation that one may roughly distinguish two zones in the resource, that are advection or diffusion dominated.

The model that couples the time evolution of the pollutant concentrations in the bioreactor $\left(S_{R}\right)$ and in the two zones ( $S_{a}$ for the active one and $S_{d}$ for the dead one) 
can be written as follows:

$$
\begin{aligned}
\dot{B} & =\mu\left(S_{R}\right) B-\frac{Q}{V_{r}} B \\
\dot{S}_{R} & =-\frac{\mu\left(S_{R}\right) B}{Y}+\frac{Q}{V_{r}}\left(S_{a}-S_{R}\right) \\
\dot{S}_{a} & =\frac{Q}{r V}\left(S_{R}-S_{a}\right)+\frac{d}{r V}\left(S_{d}-S_{a}\right) \\
\dot{S}_{d} & =\frac{d}{(1-r) V}\left(S_{a}-S_{d}\right)
\end{aligned}
$$

where $B$ represents the biomass concentration inside the bioreactor of volume $V_{r}$. The flow rate $Q$ is to be manipulated and $r \in(0,1)$ represents the ratio of the volume of the active patch over the total volume $V$ of the water resource. Finally $d$ is the diffusion rate of the pollutant between the two zones.

The first two equations comes from the classical (perfectly mixed) chemostat model (see a.e. [SW95]), where $\mu(\cdot)$ and $Y$ are respectively the growth rate function and the yield conversion factor, that are both specific to the bacterial strain that grows in the bioreactor. In the third equation we consider that the water returns to the lake with the same flow rate $Q$, assuming a perfect separation of the biomass at the output of the bioreactor without diminishing the flow rate.

We shall assume that the growth rate $\mu(\cdot)$ is monotonic, and define the usual breakeven concentration as follows:

$$
\lambda(D)=\mid \begin{array}{ll}
+\infty & \text { if }\{S>0 \text { s.t. } \mu(S)>D\}=\emptyset \\
\inf \{S>0 \text { s.t. } \mu(S)>D\} & \text { otherwise }
\end{array}
$$

A typical instance of monotonic growth rate function is given by the Monod expression

$$
\mu(S)=\frac{\mu_{\max } S}{K_{s}+S} .
$$

It is worth to be underlined that we assume that no biomass goes to the resource, i.e. that there is a perfect biomass/substrate separation at the output of the bioreactor. When the volume of the bioreactor $V_{r}$ is very small compared to the volume $r V$ of the active zone, the two first equations can be approximated by the quasi-stationary stable state $\left(B^{e q}, S_{R}^{e q}\right)$ that is defined by the equations

$$
S_{R}^{e q}(Q)=\min \left(S_{a}, \lambda\left(Q / V_{r}\right)\right), \quad B^{e q}(Q)=Y\left(S_{a}-S_{R}^{e} q(Q)\right) .
$$

The interested reader is referred to [SW95] for the mathematical analysis of the dynamics and equilibria of the chemostat model. The simple model is thus

$$
\begin{aligned}
& \dot{S}_{a}=\frac{Q}{r V}\left(S_{R}^{e q}(Q)-S_{a}\right)+\frac{d}{r V}\left(S_{d}-S_{a}\right) \\
& \dot{S}_{d}=\frac{d}{(1-r) V}\left(S_{a}-S_{d}\right)
\end{aligned}
$$

When the flow rate $Q$ is larger than $\bar{Q}\left(S_{a}\right)=V_{r} \mu\left(S_{a}\right)$, one has $S_{R}^{e q}=S_{a}$ which amounts to state that the bioreactor is not converting any substrate at all for those 
values of $Q$ (washout regime). Such situations are clearly not of interest. So the flow rate $Q$ will be chosen in the interval $\left[0, \bar{Q}\left(S_{a}\right)\right]$ and the optimal control problem can be stated as follows. For a given threshold $\underline{S}>0$ as an objective for the pollutant concentration to be reached in the resource and an initial condition $\left(S_{a}^{0}, S_{d}^{0}\right) \in \mathbb{R}_{+}^{2}$, we look for a time varying flow rate $Q(\cdot)$ that realizes the minimization

$$
T_{\bar{S}}^{\star}\left(S_{a}^{0}, S_{d}^{0}\right):=\inf _{Q(\cdot) \in \mathcal{Q}\left(S_{a}^{0}, S_{d}^{0}\right)} T_{\bar{S}}\left(S_{a}^{0}, S_{d}^{0}, Q(\cdot)\right)
$$

where $T_{\bar{S}}(\cdot)$ is the time to reach the target:

$$
T_{\bar{S}}\left(S_{a}^{0}, S_{d}^{0}, Q(\cdot)\right)=\inf \left\{t \geq 0 \mid S_{a}^{\left(S_{a}^{0}, S_{d}^{0}, Q(\cdot)\right)}(t) \leq \underline{S} \text { and } S_{d}^{\left(S_{a}^{0}, S_{d}^{0}, Q(\cdot)\right)}(t) \leq \underline{S}\right\} .
$$

Here, $S_{a}^{\left(S_{a}^{0}, S_{d}^{0}, Q(\cdot)\right)}(\cdot)$ and $S_{d}^{\left(S_{a}^{0}, S_{d}^{0}, Q(\cdot)\right)}(\cdot)$ denote the solution of 2.3$)$ with initial condition $\left(S_{a}^{0}, S_{d}^{0}\right)$ and time varying control $Q(\cdot)$. Optimal controls $Q(\cdot)$ are sought in the set

$$
\begin{aligned}
& \mathcal{Q}\left(S_{a}^{0}, S_{d}^{0}\right)=\left\{Q: \mathbb{R}_{+} \mapsto \mathbb{R}_{+}\right. \text {measurable s.t. } \\
& \left.\left.Q(t) \leq \bar{Q}\left(S_{a}^{\left(S_{a}^{0}, S_{d}^{0}, Q(\cdot)\right)}(t)\right)\right), \forall t \in\left[0, T_{\bar{S}}\left(S_{a}^{0}, S_{d}^{0}, Q(\cdot)\right)\right]\right\} .
\end{aligned}
$$

As $\mu(\cdot)$ is monotonic, there is a one to one correspondence between the flow rate $Q$ and the steady state value $S_{R}^{e q}$, as long as $Q$ is chosen in $\left[0, Q\left(S_{a}\right)\right]$ or equivalently $S_{R}^{e q}$ is chosen in the interval $\left[0, S_{a}\right]$. Consequently, we can consider that choosing the variable $Q$ in the interval $\left[0, \bar{Q}\left(S_{a}\right)\right]$ is equivalent to choosing the steady-state value $S_{R}^{e q}$ of the bioreactor in the interval $\left[0, S_{a}\right]$. This property allows a simple characterization of an optimal control strategy as a state feedback given by the following Proposition.

Proposition 2.1. Assume that the growth rate $\mu(\cdot)$ is a $C^{1}$ concave increasing function with $\mu(0)=0$.

$i$. For each positive number $S_{a}$, the function

$$
S_{R}^{e q} \mapsto \gamma\left(S_{R}^{e q}, S_{a}\right):=\mu\left(S_{R}^{e q}\right)\left(S_{a}-S_{R}^{e q}\right)
$$

admits an unique maximum on $\left[0, S_{a}\right]$, denoted $S_{R}^{e q \star}\left(S_{a}\right)$, and the map

$$
\Phi: S_{a} \mapsto \Phi\left[S_{a}\right]=V_{r} \mu\left(S_{R}^{e q \star}\left(S_{a}\right)\right)
$$

is $C^{1}$.

ii. The $S_{a}$-feedback $\Phi$ solves the minimal time control problem (and guarantees that the minimal time function is finite for any initial condition in $\left.\mathbb{R}_{+}^{2}\right)$.

One can check that for any $S_{a}>0$ the function $S_{R}^{e q} \mapsto \gamma\left(S_{R}^{e q}, S_{a}\right)$ is non-negative continuous on $\left[0, S_{a}\right]$, null at the boundary of this interval, and thus admits a maximum. Furthermore, one can easily verify that it is concave when the function $\mu(\cdot)$ is increasing concave, guaranteeing then the uniqueness of the maximizer $S_{R}^{e q \star}\left(S_{a}\right)$. As the function $\gamma$ is $C^{1}$ w.r.t. to both arguments and the maximizer $S_{R}^{e q \star}\left(S_{a}\right)$ is unique for each $S_{a}$, Danskin's Theorem (see [Dan66]) provides then the $C^{1}$ regularity of the map $\Phi$. Moreover, one can check that the usual Monod law given in 2.2 is a concave function, that leads to the following expression of the function $S_{R}^{e q \star}$ :

$$
S_{R}^{e q \star}\left(S_{a}\right)=\sqrt{K_{s}^{2}+K_{s} S_{a}}-K_{s} .
$$


In [RRR15, it is also shown that this feedback is robust w.r.t. the approximation of the quasi-steady state of the dynamics 2.1a)-2.1b).

The open-loop representation of this feedback is given by $Q(t)=\Phi\left[S_{a}(t)\right]$ where $S_{a}($. is the solution of equations $2.3 \mathrm{a}-2.3 \mathrm{~b}$ where $Q$ is replaced by $\Phi\left[S_{a}\right]$.

REMARK 1. This result has been first proved in GHR11] for a single homogeneous zone (i.e. $r=1$ ) and later extended in GRRR12 for the two patches model. The solution of the optimization problem on this ODE dynamics possesses the remarkable feature that the optimal feedback given in Proposition 2.1 is independent of the parameters $r$ and $d$ (although the optimal trajectory and the minimal time do depend on it). Thus, the implementation of the optimal feedback can be done without the knowledge of the heterogeneity characteristics $(r, d)$ of the two patches model, and stays valid even if one consider that the parameters $(r, d)$ could be time varying. It simply needs the online measurement of the concentration in the "active" zone, that is where the water is extracted from the resource.

2.2. Feedback control applied to the PDEs model. The nice property of feedback $\Phi$, mentioned in Remark 1 is that $\Phi$ is independent of the parameters $(d, r)$. This has led us to consider the application of this feedback on a PDE model of time and space evolution of pollutant in the lake, based on the Navier-Stokes equations. We have also considered that the concentration $S_{a}$ of the "active" zone that is required by this feedback should correspond to the concentration $S_{\text {out }}(\cdot)$ at the pumping location on the boundary of the water resource.

Considering a time-varying control $Q($.), we allow the pollution concentration in the resource to depend both on time and space ${ }^{*} S_{L}=S_{L}(t, \mathbf{x})$ where $\mathbf{x} \in \mathbb{R}^{2}$.

Let $\Omega \cup \mathbb{R}^{2}$ be the water resource with boundary $\partial \Omega=\Gamma_{0} \cup \Gamma_{\text {in }} \cup \Gamma_{\text {out }}$, where $\Gamma_{\text {in }}$ (resp. $\Gamma_{\text {out }}$ ) holds for the part of the resource border where the inflow (resp. outflow) pump is installed, and $\Gamma_{0}$ is the rest of the border (see Figure 2.3. .

The evolution of the pollutant concentration $S_{L}(t, \mathbf{x})$ in the resource is modeled by the following advection-diffusion equation:

$$
\frac{\partial S_{L}}{\partial t}+\mathbf{U} \cdot \nabla S_{L}-\nu_{S} \Delta S_{L}=0
$$

where $\nu_{S}$ is the pollutant diffusivity, and $\mathbf{U}(t, \mathbf{x})=(u(t, \mathbf{x}), v(t, \mathbf{x}))$ is the fluid velocity in the resource, computed thanks to the Navier-Stokes equations (the reference model for incompressible viscous fluids, see [Tem01]):

$$
\begin{aligned}
\frac{\partial \mathbf{U}}{\partial t}+\mathbf{U} \cdot \nabla \mathbf{U}+\nabla p-\nu_{u} \Delta \mathbf{U} & =0, \\
\nabla \cdot \mathbf{U} & =0 .
\end{aligned}
$$

Here, $p$ stands for the pressure exerted on the fluid, and $\nu_{u}$ denotes the water viscosity. Naturally, equations (2.5) and 2.6) are supplemented with initial conditions (fluid at rest with initial pollution)

\footnotetext{
*For the sake of simplicity, we assume that the pollution is driven by a $2 \mathrm{D}$ flow (at the surface of the water resource).
} 


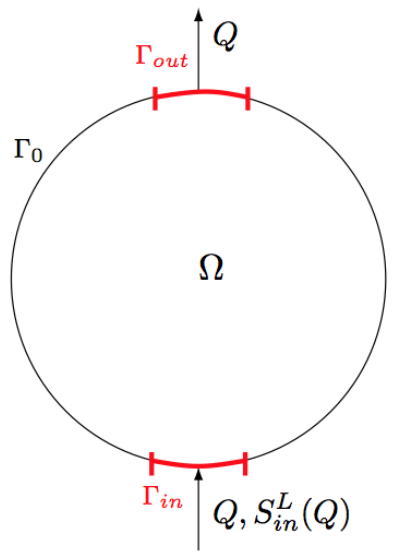

Fig. 2.3: Water resource $\Omega$ with boundary $\partial \Omega=\Gamma_{\text {in }} \cup \Gamma_{\text {out }} \cup \Gamma_{0}$. For the sake of readability, the size of inflow and outflow regions have been artificially increased.

$$
\begin{aligned}
S_{L}(t=0, \mathbf{x}) & =S_{0}(\mathbf{x}) \quad \forall \mathbf{x} \in \Omega, \\
\mathbf{U}(t=0, \mathbf{x}) & =0 \quad \forall \mathbf{x} \in \Omega,
\end{aligned}
$$

and boundary conditions on $\partial \Omega$. On the inflow boundary, the water velocity scales with the flow rate $Q(t)$ and the pollutant concentration in the bioreactor. Under the assumption that the volume $V_{r}$ of the bioreactor is small compared to the volume of the lake, the time scale of the bioreactor dynamics, that is given by the dilution rate $Q(t) / V_{r}$, is large compared to the time scale of the evolution of the pollutant concentration in the lake. Moreover, the time evolution of the input flow $Q(t)$ being expressed as a feedback on the output concentration of the lake, is also small compared to the bioreactor dynamics. Exactly as in the o.d.e. based modeling (see Section 2.1), we thus consider the quasi-stationary approximation of the dynamics of the bioreactor, that provides a pollutant concentration of the bioreactor equal to $S_{R}^{e q}(Q(t))$. We have then on $\Gamma_{i n}$ :

$$
\begin{aligned}
\mathbf{U}(t, \mathbf{x})=Q(t) \times \mathbf{U}_{\mathbf{i n}}(\mathbf{x}) & \forall \mathbf{x} \in \Gamma_{i n} \\
S_{L}(t, \mathbf{x})=S_{R}^{e q}(Q(t)) & \forall \mathbf{x} \in \Gamma_{i n} .
\end{aligned}
$$

On the outflow boundary $\Gamma_{\text {out }}$ the boundary conditions are:

$$
\begin{aligned}
\mathbf{U}(t, \mathbf{x})=Q(t) \times \mathbf{U}_{\text {out }}(\mathbf{x}) & \forall \mathbf{x} \in \Gamma_{\text {out }} \\
\frac{\partial S_{L}}{\partial n}(t, \mathbf{x})=0 & \forall \mathbf{x} \in \Gamma_{\text {out }}
\end{aligned}
$$

Finally, on the rest of the resource border, we have: 


$$
\begin{aligned}
\mathbf{U}(t, \mathbf{x}) & =0 & \forall \mathbf{x} \in \Gamma_{0}, \\
\frac{\partial S_{L}}{\partial n}(t, \mathbf{x}) & =0 & \forall \mathbf{x} \in \Gamma_{0} .
\end{aligned}
$$

The functions $\mathbf{U}_{\text {in }}$ and $\mathbf{U}_{\text {out }}$ are unitary parabolic functions that describe the velocity profile at the boundary (Poiseuille flow);

$$
\begin{array}{r}
\mathbf{U}_{\text {in }} \cdot \vec{\tau}=\mathbf{U}_{\text {out }} \cdot \vec{\tau}=0, \\
-\int_{\Gamma_{\text {in }}} \mathbf{U}_{\text {in }} \cdot \vec{n} d \Gamma=\int_{\Gamma_{\text {out }}} \mathbf{U}_{\text {out }} \cdot \vec{n} d \Gamma=1,
\end{array}
$$

where $\vec{n}(\mathbf{x})$ is the unitary vector, orthogonal to $\partial \Omega$ in $\mathbf{x}$, pointing outward, and $\vec{\tau}(\mathbf{x})$ provide the tangential direction.

The time-depending flow rate $Q$ in equations $2.8 \mathrm{a}$ and $2.9 \mathrm{a}$ corresponds to the inflow/outflow discharge in the bioreactor (see Figure 2.4) so that the bioreactor and resource volumes are conserved at all times.

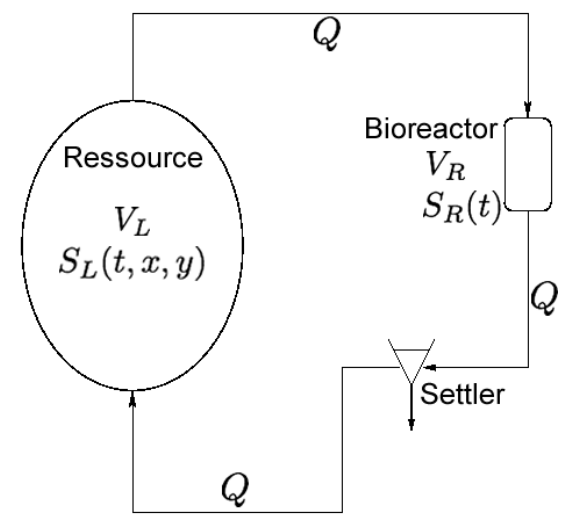

Fig. 2.4: Schematic view of the resource and biorector. When modeled by the couple PDE-ODE system, $S_{L}$ is a function of time and space.

Given a time varying control $Q($.$) , we consider S_{L}($.$) and U($.$) solutions of equations$ (2.5) and 2.6) with initial conditions (2.7) and boundary conditions (2.8)-(2.10); we define the output concentration $S_{\text {out }}$ (pumped from the lake into the bioreactor) as

$$
S_{\text {out }}(t)=\frac{1}{\left|\Gamma_{\text {out }}\right|} \int_{\Gamma_{\text {out }}} S_{L}(t, x) d x
$$

This allows the definition of an operator

$$
\begin{aligned}
\Theta: \mathcal{C}^{1}\left(\mathbb{R}_{+}, \mathbb{R}_{+}\right) & \rightarrow \mathcal{C}^{1}\left(\mathbb{R}_{+}, \mathbb{R}_{+}\right) \\
Q(.) & \mapsto \Theta(Q(.))(.)=S_{\text {out }}(.),
\end{aligned}
$$

where $S_{\text {out }}($.$) is defined in 2.12$. Applying a feedback strategy $S_{\text {out }} \mapsto \Phi\left[S_{\text {out }}\right]$ awaits to consider a fixed point of $\Phi \circ \Theta$, i.e. a control $Q^{*}($.$) that satisfies$

$$
\Phi\left[\Theta\left(Q^{*}(.)\right)(t)\right]=Q^{*}(t), \quad \forall t>0
$$


which amounts to require the associated $S_{\text {out }}($.$) to fulfill \Phi\left[S_{\text {out }}(t)\right]=Q^{*}(t)$ at any time $t$. In practice, we approximate $Q^{*}$ by computing a time-discretization of step $\Delta t$ with piecewise constant control $Q$ : given $S_{\text {out }}(k \Delta t)(k=0, \cdots)$, we compute $Q_{k}^{*}=\Phi\left[S_{\text {out }}(k \Delta t)\right]$ to be applied as a constant control on the interval $[k \Delta t,(k+1) \Delta t)$.

2.3. Calibration of the ODEs model on simulations of the PDEs model. The simple two patches model proposed in Section 2.1 relies on two parameters $r, d$ that roughly describe heterogeneity in the resource. For a given initial distribution $S_{0}(\cdot)$ of pollutant in the resource, that we have supposed to be uniform: $S_{0}(\cdot) \equiv S_{0}$, we look for a couple $(r, d)$ such that the solution $S_{a}(\cdot)$ of the ODEs model 2.3a)-(2.3b) with feedback 2.4 and initial condition $S_{a}^{0}=S_{d}^{0}=S_{0}$ is as closed as possible, in $L^{2}$ norm, to the output $S_{\text {out }}(\cdot)$ of the solution of the PDEs model with feedback (2.4) applied to $S_{\text {out }}$ (see Section 2.2):

$$
\min _{(r, d)} \int_{0}^{T_{\bar{S}}^{\star}\left(S_{0}, S_{0}\right)}\left(S_{\text {out }}(t)-S_{a}(t)\right)^{2} d t .
$$

The objective is to verify that the two patches model, after being optimized, can give a good approximation of the output of the PDEs model, and moreover can serve as a good prediction tool for the time necessary to treat the pollution with the proposed feedback.

3. Numerical simulations. We now introduce some numerical simulations performed both with the complete PDE-ODE model, and with the simple ODE models. All simulations are ran with a constant flow rate $Q=1$ so that there is no feedback strategy here. This objective of this section is simply to compare the 3 different models.

3.1. The reference PDE-based model. We consider here the bioremediation model made of equations 2.1a)-(2.1b coupled with the PDEs 2.5)-(2.6). These equations are supplemented with initial conditions (2.7) and boundary conditions 2.8)-2.10 where $Q(t)$ is taken constant. Numerical simulations are conducted with the finite element method implemented with Freefem ++ (see [HPLH04]). Figure 3.1 below indicates the evolution of the pollutant concentration in the resource. At $t=0$, the fluid is at rest and the pollution is homogeneous (see Figure 3.1a). Figures 3.1b illustrates the fact that the pollution does not decrease the same way in each and every location of the resource (hence the dead/active zone model).

3.2. ODE models. We now replace the PDE-ODE coupled system used in Section 3.1 by the (simpler) system of ODEs 2.1).

3.2.1. The single zone model. With $d=0$ and $r=1$, the model described by equations (2.1) corresponds to the single zone model first considered in GHR11. It is not surprising to figure out with Figure 3.2 that this simple model does not reproduce satisfactorily the bioremediation of the polluted resource. Clearly, this model cannot handle spatial heterogeneity in the resource as the PDE model does.

3.2.2. The two zones model. To improve the single zone model, we optimize the values of $r \in(0,1)$ and $d>0$ in order to better approximate the reference numerical solution obtained with the complete Navier-Stokes equations. Figure 3.3 illustrates the fact that the two-zones model (with appropriate values of $r$ and $d$ ) is a much more accurate approximation of the reference model than the single zone one 


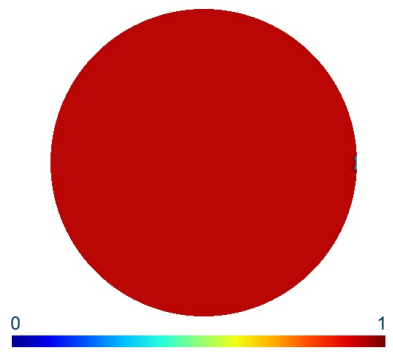

(a) Pollutant concentration at $t_{0}=0$.

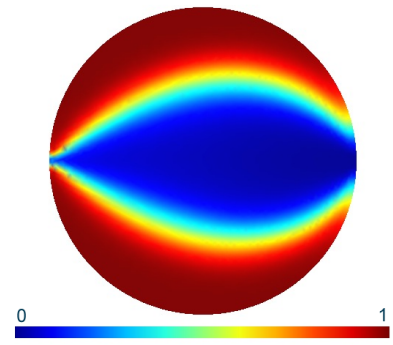

(b) Pollutant concentration at $t_{1}>0$.

Fig. 3.1: Evolution of the (renormalized) pollutant concentration in the resource thanks to the coupled PDE-ODE system. The water is extracted from the left side of the domain, treated in the bioreactor and injected back through the right side.

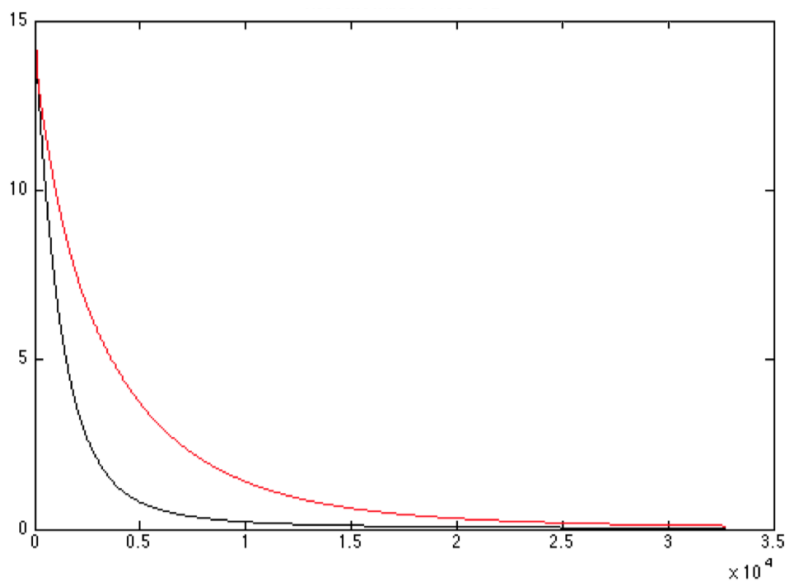

Fig. 3.2: Time-evolution of the pollutant concentration in the resource with uniform initial value $S_{0}=15$. Reference PDE model (upper red curve) and single zone model (lower black curve).

(corresponding to $r=1, d=0$ ).

REMARK 2. In the simulations performed in Section 3.2 we need to choose a diffusivity coefficient $\nu_{S}$ for the pollutant (see Equation 2.5). It is interesting to notice (see Figure 3.4 that the behavior of the best coefficient $d$ (so that the simple model correctly approximates the reference model) is monotonic: we understand this as an illustration of the "diffusion" role of $d$ between the active and dead zones.

4. Conclusion. In this work we have compared two simple models given by systems of ordinary differential equations calibrated with a reference PDE model based on the Navier-Stokes equations for the resource hydrodynamics. Whereas the first single zone model cannot reproduce satisfactorily the spatial heterogeneity of pollutant in the lake, the calibration of the second one (with a dead zone and an 


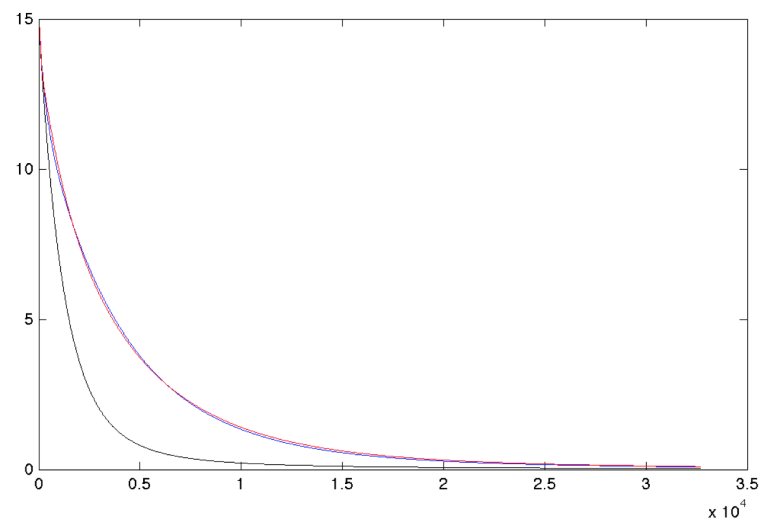

Fig. 3.3: Time-evolution of the pollutant concentration in the resource with uniform initial value $S_{0}=15$. Reference PDE model (upper red), single zone model (lower black) and two zones model (upper blue). Parameters: $\nu_{S}=5.10^{-3}$, $(r, d)=(0.346,0.228)$. The blue and red curves are indistinguishable.

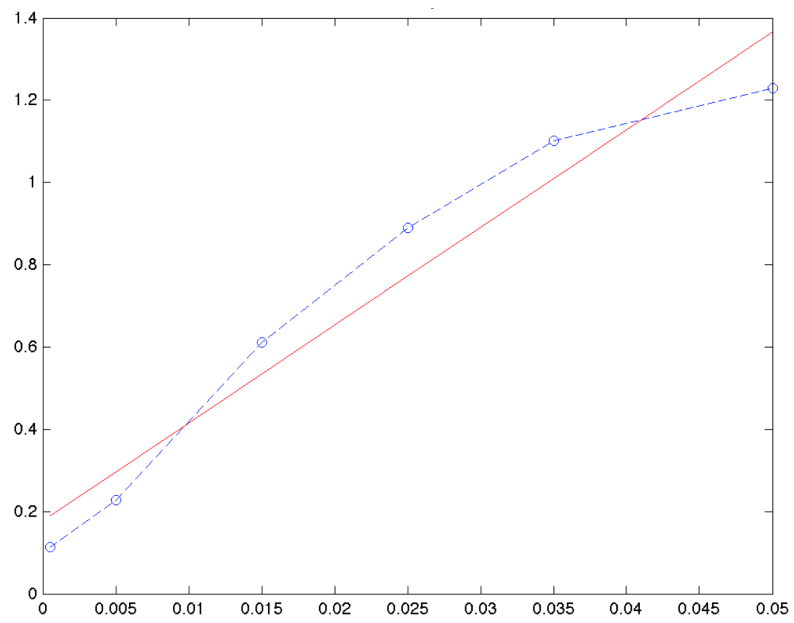

Fig. 3.4: Diffusion parameter $d$ versus viscosity $\nu_{S}$ (dashed blue). Best mean-square fit (plain red).

active one) provides much better approximation of the reference model. This two zones model is indeed more sophisticated that the first one, but is way simpler than the Navier-Stokes equations.

Thanks to previous works on optimal control, a minimal time strategy can be derived from the two-zones model that can then be applied to the reference model. As shown formerly, the optimal strategy that provides the on-line adjustment of the flow rate for minimizing the total decontamination time requires the single on-line measurement of the pollutant concentration at the output of the lake, that is provided by the reference model.

Finally, we have proposed in this work an original scheme that provides 
- a simple and easily applicable feedback control of the flow rate,

- simple approximations of the input-output dynamics of the pollutant concentration of the lake and the expected minimal time of decontamination (with a system of two ODEs that offers quite light computation times),

thanks to the combination of numerical simulations of a PDE based model of the hydrodynamics in the lake, and a calibrated system of ODEs for given geometrical data of the lake and diffusivity properties of the pollutant. Even better approximations with higher dimensions of the ODE models (i.e. considering more than two zones) could be obtained with the same methodology.

This approach with feedback control on simple models provide bases for future efficient decision making tools for the bioremediation of natural water reservoirs.

Acknowledgments. The authors were supported by the research programs LEFEINSU CoCoA, LabEx NUMEV and Inria associated team DYMECOS. The authors also want to thank Vincent Guinot and Jérôme Harmand for fruitful discussions related to this work.

\section{REFERENCES}

[Alf06]

[BBCMA03]

[Dan66]

[DGK72]

[EPD09]

[GDG71]

[GHR11]

[GRRR12]

[GvD02]

[HPLH04]

[KHO0]

[Rav00]

[RRR15]

$\left[\mathrm{SJL}^{+} 07\right]$

[SKLB08]
J. Alford. Bioprocess control: Advances and challenges. Computers and Chemical Engineering, 30:1464-1475, 2006.

J. Banga, E. Balsa-Canto, C. Moles, and A. Alonso. Dynamic optimization of bioreactors: a review. Proceedings of the Indian Academy of Sciences, 69:257-265, 2003.

J.M. Danskin. The Theory of Max-Min, with Applications. SIAM Journal on Applied Mathematics, 14:641-664, 1966.

G. D'Ans, D. Gottlieb, and P. Kokotovic. Optimal control of bacterial growth. Automatica, 8:729-736, 1972.

V. Estrada, E.R. Parodi, and M. S. Diaz. Addressing the control problem of algae growth in water reservoirs with advanced dynamic optimization approaches. Computers and Chemical Engineering, 33:2063-2074, 2009.

P. Kokotovic G. D'Ans and D. Gottlieb. Time-optimal control for a model of bacterial growth. J. Optim. Theory Appl., 7:61-69, 1971.

P. Gajardo, J. Harmand, and A. Rapaport. Minimal time bioremediation of natural water resources. Automatica, 47(8):6-6, July 2011.

P. Gajardo, H. Ramirez, V. Riquelme, and A. Rapaport. Bioremediation of natural water resources via optimal control techniques. In R. Mondaini, editor, BIOMAT 2011: International Symposium on Mathematical and Computational Biology, pages 178-190. World Scientific, 2012.

R.D. Gulati and E. van Donk. Lakes in the netherlands, their origin, eutrophication and restoration: state of the art reviews. Hydrobiologia, 478:73-106, 2002.

F. Hecht, O. Pironneau, and A. Le Hyaric. FreeFem++ manual. 2004.

P. Kittisupakorn and M. Hussain. Comparison of optimisation based control techniques for the control of a cstr. International Journal of Computer Applications in Technology, 13(3-5):178-184, 2000.

S. S. Ravindran. A reduced-order approach for optimal control of fluids using proper orthogonal decomposition. International Journal for Numerical Methods in Fluids, 34(5):425-448, 2000.

H. Ramirez, A. Rapaport, and V. Riquelme. Optimal feedback synthesis and minimal time function for the bioremediation of water resources with two patches. Preprint hal-01069581 https://hal.archives-ouvertes.fr/ hal-01069581, 2015.

M. Sondergaard, E. Jeppesen, T.L. Lauridsen, C. Skov, E.H. van Nes, R. Roijackers, E. Lammens, and R. Portielje. Lake restoration: successes, failures and long term effects. Journal of Applied Ecology, 44:1095-1105, 2007.

A. Soukkou, A. Khellaf, S. Leulmi, and K. Boudeghdegh. Optimal control of a cstr process. Braz. J. Chem. Eng, 25(4):799-812, 2008. 
[Spi04] D. Spitzer. Maximization of steady-state bacterial production in a chemostat with ph and substrate control. Biotechnology and Bioengineering, 18(2):167-178, 2004.

[SW95] H. L. Smith and P. Waltman. The theory of the chemostat, volume 13 of Cambridge Studies in Mathematical Biology. Cambridge University Press, Cambridge, 1995.

[Tem01] R. Temam. Navier-Stokes equations. AMS Chelsea Publishing, Providence, RI, 2001. Theory and numerical analysis, Reprint of the 1984 edition. 


\section{Appendix A. Links between PDE and ODE models.}

In this appendix we start from the transport-diffusion equation $(2.5)$ and justify the simplified equations $(2.1 \mathrm{c})$ and $(2.1 \mathrm{~d})$. Let us recall equation (2.5):

$$
\frac{\partial S_{L}}{\partial t}+\mathbf{U} \cdot \nabla S_{L}-\nu_{S} \Delta S_{L}=0
$$

We define the averaged pollutant concentration over the domain:

$$
\overline{S_{L}}(t)=\frac{1}{|\Omega|} \int_{\Omega} S_{L}(t, x, y) d x d y .
$$

Integrating equation A.1 over the whole domain $\Omega$, we have:

$$
\frac{d \overline{S_{L}}}{d t}+\frac{1}{|\Omega|} \int_{\Omega} \mathbf{U}(t, x, y) \cdot \nabla S_{L}(t, x, y) d x d y-\frac{\nu_{S}}{|\Omega|} \int_{\Omega} \Delta S_{L}(t, x, y) d x d y=0 .
$$

Thanks to the free divergence of $\mathbf{U}$ and to boundary conditions $2.8,2.9$, and 2.10 , we obtain:

$$
\frac{d \overline{S_{L}}}{d t}-\frac{1}{|\Omega|} \int_{\Gamma_{\text {in }}} \frac{Q S_{L}}{\left|\Gamma_{\text {in }}\right|} d s+\frac{1}{|\Omega|} \int_{\Gamma_{\text {out }}} \frac{Q S_{L}}{\left|\Gamma_{\text {out }}\right|} d s-\frac{\nu_{S}}{|\Omega|} \int_{\Gamma_{\text {in }}} \frac{\partial S_{L}}{\partial n} d s=0,
$$

where $n$ classically denotes the outward-pointing vector normal to $\Omega$ on $\partial \Omega$.

We now replace the boundary condition $2.8 \mathrm{a}$ on $\Gamma_{i n}$ by the following Robin conditiont:

$$
-\nu_{S} \frac{\partial S_{L}}{\partial n}=\frac{Q}{\left|\Gamma_{i n}\right|}\left(S_{L}-S_{i n}\right)
$$

and obtain:

$$
\frac{d \overline{S_{L}}}{d t}-\frac{Q}{|\Omega|} S_{\text {in }}+\frac{Q}{|\Omega|\left|\Gamma_{\text {out }}\right|} \int_{\Gamma_{\text {out }}} S_{L} d s=0
$$

Now, assuming that the average concentration close to $\Gamma_{\text {out }}$ is the same as the average concentration in the whole domain (in particular this is true if one assumes that $S_{L}$ does not depend on space), then we have:

$$
\frac{d \overline{S_{L}}}{d t}=\frac{Q}{|\Omega|}\left(S_{i n}-\overline{S_{L}}\right)
$$

which is exactly the one zone model.

Now, if we consider (as it can be inferred from Figure 2.2) that we have two zones in the lake (the blue one $\Omega_{1}$ and the red one $\Omega_{2}$ ) separated by an interface $\Gamma_{1 / 2}$ on which we have $\mathbf{U} \cdot n_{1}=0$ where $n_{1}$ is the outward-pointing vector normal to $\Omega_{1}$ on $\Gamma_{1 / 2}$, we can restart from equation (A.1) but integrate it over the active (blue) zone $\Omega_{1}$ only, and obtain after the same kind of computation:

$$
\frac{d S_{1}}{d t}-\frac{Q}{\left|\Omega_{1}\right|} S_{\text {in }}+\frac{Q}{\left|\Omega_{1}\right|\left|\Gamma_{\text {out }}\right|} \int_{\Gamma_{\text {out }}} S_{L} d s-\frac{\nu_{S}}{\left|\Omega_{1}\right|} \int_{\Gamma_{1 / 2}} \frac{\partial S_{L}}{\partial n_{1}} d s=0
$$

\footnotetext{
${ }^{\dagger}$ In the case where the viscosity coefficient $\nu_{S}$ is zero (or very small), these boundary conditions are equivalent (or similar).
} 
where $S_{1}$ is the averaged pollutant concentration in the active zone $\Omega_{1}$ :

$$
S_{1}(t)=\frac{1}{\left|\Omega_{1}\right|} \int_{\Omega_{1}} S_{L}(t, x, y) d x d y .
$$

Assuming again than the average concentration close to $\Gamma_{\text {out }}$ is the same as the average concentration in $\Omega_{1}$, we have:

$$
\frac{d S_{1}}{d t}=\frac{Q}{\left|\Omega_{1}\right|}\left(S_{i n}-S_{1}\right)+\frac{\nu_{S}}{\left|\Omega_{1}\right|} \int_{\Gamma_{1 / 2}} \frac{\partial S_{L}}{\partial n_{1}} d s,
$$

Considering now the dead zone $\Omega_{2}$ with the outward-pointing vector normal $n_{2}=-n_{1}$ on $\Gamma_{1 / 2}$, the same computations lead to:

$$
\frac{d S_{2}}{d t}=\frac{\nu_{S}}{\left|\Omega_{2}\right|} \int_{\Gamma_{2 / 1}} \frac{\partial S_{L}}{\partial n_{2}} d s,
$$

with

$$
S_{2}(t)=\frac{1}{\left|\Omega_{2}\right|} \int_{\Omega_{2}} S_{L}(t, x, y) d x d y .
$$

We now consider that the integral of the normal derivative of $S_{L}$ along the interface $\Gamma_{1 / 2}$ scales like $S_{2}-S_{1}$, that is there exists a positive constant $K$ such that

$$
\int_{\Gamma_{1 / 2}} \frac{\partial S_{L}}{\partial n_{1}} d s=K\left(S_{2}-S_{1}\right) .
$$

Finally, considering equations (A.6) and A.7) in which the integral is replaced by the above approximation, we indeed obtain the two ODEs $(2.1 \mathrm{c})$ and $(2.1 \mathrm{~d})$. 